\title{
Mathematical Modelling and Analysis of Memristors with and without its Temperature Effects
}

\author{
Bhavani Prasad, Kamaraju Maddu, and Venkata Lakshmi
}

\begin{abstract}
In this paper the main goal is to study the principle structure and characteristics of single and multiple memristors and also the temperature effects. The complete analysis described here is done by using matlab Simulink. The relationship between the on resistance, off resistance and ionic mobility with respect to temperature has been analyzed and shown graphically. The memristor can be used as a High speed switch and it can be used in non volatile computer memories due to its higher switching speeds.
\end{abstract}

Keywords-Charge, flux, Memristance, Memristor, Temperature

\section{INTRODUCTION}

$I^{1}$ 1971, an American scientist Leon Chua proposed a new element called memristor [1]. It is a combination of memory and resistor. Stanley Williams of Hewlett Packard invented a first physical prototype in 2008. It is based on nonlinearity relationship between charge and flux. The property of this nonlinear element is to memorize the amount of charge which has passed through it [2].

The prototype is made of titanium dioxide [3, 4]. The schematic diagram of memristor is given in fig 1 [3]. A layer of titanium dioxide is placed in between two platinum electrodes. This layer has two sub layers. Oxygen vacancies are doped to the layer which is called the first sub layer. The electrical resistance of this layer is small. The layer which consists of pure titanium dioxide is another sub layer which has high specific electrical resistance. The width of the doped layer is $w$ and width of the whole memristor is $\mathrm{D}$.

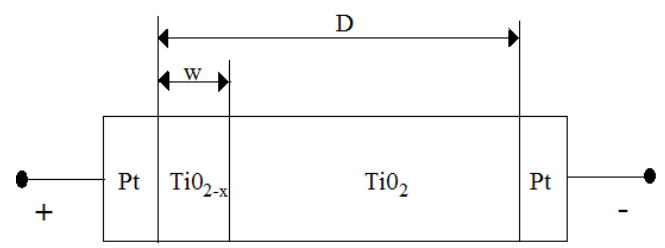

Fig. 1. Schematic of titanium dioxide memristor

The initial width of the doped layer is $w=1 \mathrm{~nm}$. The width of the whole memristor is $D=10 \mathrm{~nm}$. The average value of ionic mobility of oxygen vacancies is $\mu_{v}$

$$
\mu_{v}=1.10^{14}\left(m^{2} / V . s\right)
$$

When the voltage is applied across the memristor oxygen vacancies starts movement. The oxygen vacancies begin to

All authors are with Gudlavalleru Engineering College, Gudlavalleru, Department of Electronics and Communications, India (e-mail: bhavaniprasad.ece@gmail.com, madduraju@yahoo.com, tvlthota@gmail. com). move from one electrode to other electrode when the potential of the electrode connected to doped layer is positive. The charges pass through the layer of pure titanium dioxide. Then the electrical resistance of the whole memristor is decreases. If the voltage source is switched off, the current flowing stops. But in the second sub layer the charges of oxygen vacancies remain enveloped. Before interrupting the voltage source the memristor retains the last value of its resistance. $R_{O N}$ is the resistance of the doped layer and $R_{O F F}$ is the resistance of the undoped layer.

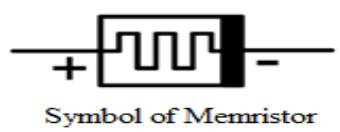

Fig. 2. Symbol of memristor

The symbol of the memristor is shown in fig 2 in which the negative end is labelled black.

\section{MATHEMATICAL ANALYSIS OF MEMRISTOR}

A simple memristor circuit with one memristor and a voltage source is shown in fig 3 [5]. e(t) is the voltage source, $i(t)$ is the current in the circuit and $\mathrm{u}(\mathrm{t})$ is the voltage drop across the memristor.

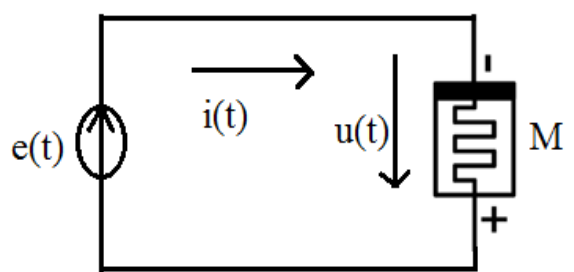

Fig. 3. A circuit with single memristor and voltage source

The flux linkage is presented as

$$
\psi(t)=\int_{0}^{t} u(t) d t
$$

The following expression is obtained when KVL (Kirchhoff's voltage law) is applied on the equivalent circuit of fig 1 when doped and undoped layers are connected in series

$$
u(t)=\left(R_{O N} \frac{w(t)}{D}+R_{O F F}\left(1-\frac{w(t)}{D}\right)\right) i(t)
$$

The rate of moving of boundary between the zones is presented as a derivative of width of doped region with respect to time and it is given as 


$$
v_{0}(t)=\frac{d w(t)}{d t}=\mu_{v} \frac{R_{O N}}{D} i(t)
$$

After integration of equation (4) with respect to time, the equation is

$$
w(t)=\mu_{v} \frac{R_{O N}}{D} q(t)
$$

The charge $\mathrm{q}(\mathrm{t})$ is substituted in the above equation (5) withquantity of charge. Then the equation is as follows

$$
Q_{D}=\frac{D^{2}}{\mu_{v} R_{O N}}
$$

The ratio of the width of doped layer to the whole width of the memristor with $\mathrm{x}(\mathrm{t})$ is

$$
x(t)=\frac{w(t)}{D}=\frac{q(t)}{Q_{D}}
$$

Where $\mathrm{x}(\mathrm{t})$ is the normalized width of the doped region.

Substituting the equation (5) in equation (3). Then the equation is

$$
M(q)=R_{O F F}\left(1-\frac{\mu_{v} R_{O N}}{D^{2}} q(t)\right)=R_{O F F}\left(1-\frac{q(t)}{Q_{D}}\right)
$$

The relation between the charge and flu is obtained by transforming the equation (4).

$$
q(t)=Q_{D}\left(1-\sqrt{1-\frac{2}{Q_{D} R_{O F F}} \psi(t)}\right)
$$

The relationship between $\mathrm{x}(\mathrm{t})$ and $\psi(\mathrm{t})$ is obtained by analogous transformation.

$$
x(t)=1-\sqrt{1-\frac{2 \mu_{D}}{r D^{2}} \psi(t)}
$$

The current is obtained by differentiating the equation (9)

$$
i(t)=\frac{u(t)}{R_{\text {OFF }} \sqrt{1-\frac{2 \mu_{D}}{r D^{2}} \int u(t) d t}}
$$

\section{DEPENDENCE OF ON RESISTANCE, OFFRRESISTANCE, MOBILITY ON TEMPERATURE}

The Simulink model is constructed for the circuit of fig. 3 by using the formula (9) and Kirchhoff's voltage law. The model is given in fig. 4 . The circuit is constructed at amplitude of $1 \mathrm{v}$, frequency $1 \mathrm{~Hz}, \mathrm{R}_{\mathrm{ON}}=100 \Omega, \mathrm{R}_{\mathrm{OFF}}=16 \mathrm{~K} \Omega$.

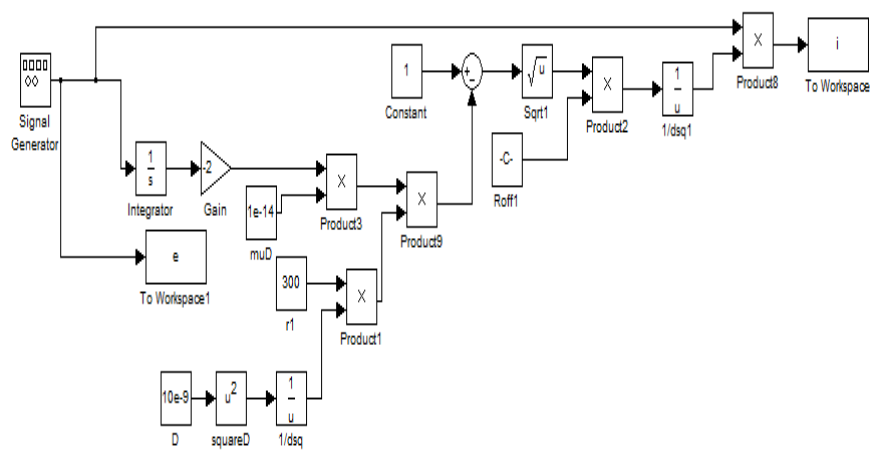

Fig. 4. Simulink model of circuit with single memristor
The resistances in on and off states of memristor are $\mathrm{R}_{\mathrm{ON}}$ and $\mathrm{R}_{\mathrm{OFF}}$. These resistances are considering with respect to temperatures in equation (12) and equation (13) [6]. The Simulink blocks for the equation (12) and equation (13) is shown in fig. 5 and fig. 6.

$$
\begin{gathered}
R_{\text {ON }}=6.33 * 10^{16} T^{-6}[\Omega] \\
R_{\text {OFF }}=2.06 * 10^{18} T^{-5.72}[\Omega]
\end{gathered}
$$

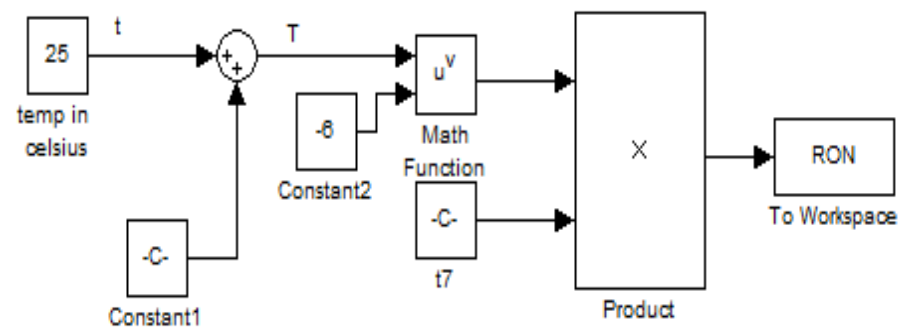

Figure 5: Simulink model for on resistance

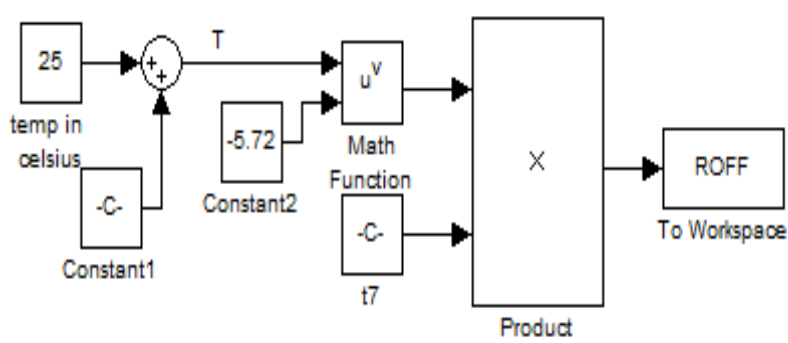

Fig. 6. Simulink model for off resistance

The mobility of oxygen vacancies with respect to temperature is given by the equation (14) [6] and the simulink block is shown in fig. 7 .

$$
\mu_{v}=1 * 10^{54}\left(-0.0005 T^{-18}+0.2394 T^{-17}\right)
$$

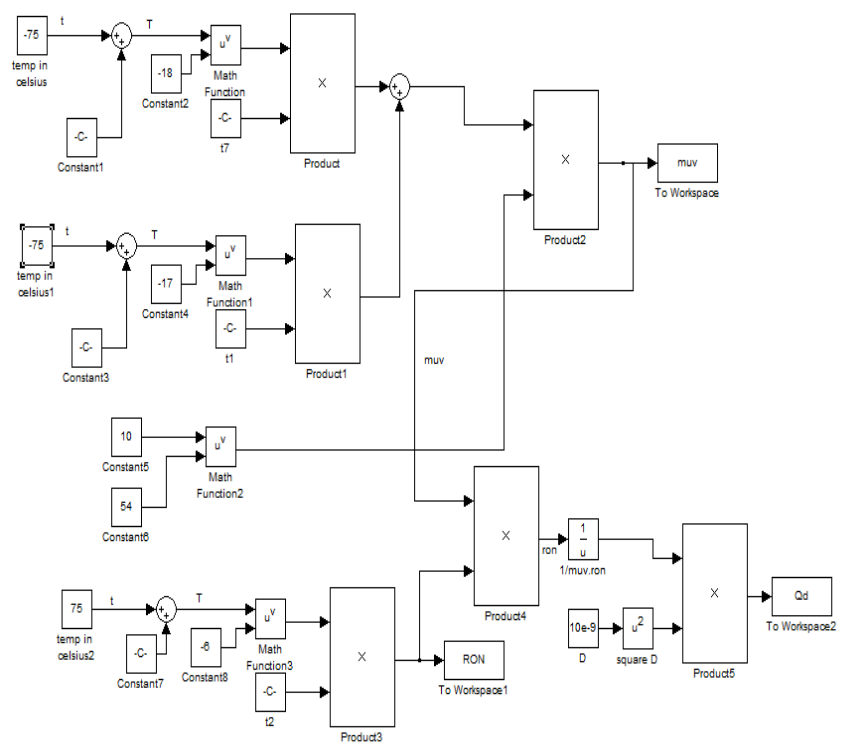

Fig. 7. Simulink model for mobility and charge

Figure 8 is the simulink block for two memristors [5] that are connected in series with different parameters $R_{O F F}$ and $R_{O N}$. For the first memristor $\mathrm{R}_{\mathrm{ON} 1}=200 \Omega$ and $\mathrm{R}_{\mathrm{OFF} 1}=60 \mathrm{~K} \Omega$ and for 
the second memristor $\mathrm{R}_{\mathrm{ON} 2}=100 \Omega$ and $\mathrm{R}_{\mathrm{OFF} 2}=16 \mathrm{~K} \Omega$. The circuit is simulated at a circular frequency of $8 \mathrm{rad} / \mathrm{sec}$ and $\mathrm{e}=2.7 \mathrm{~V}$.

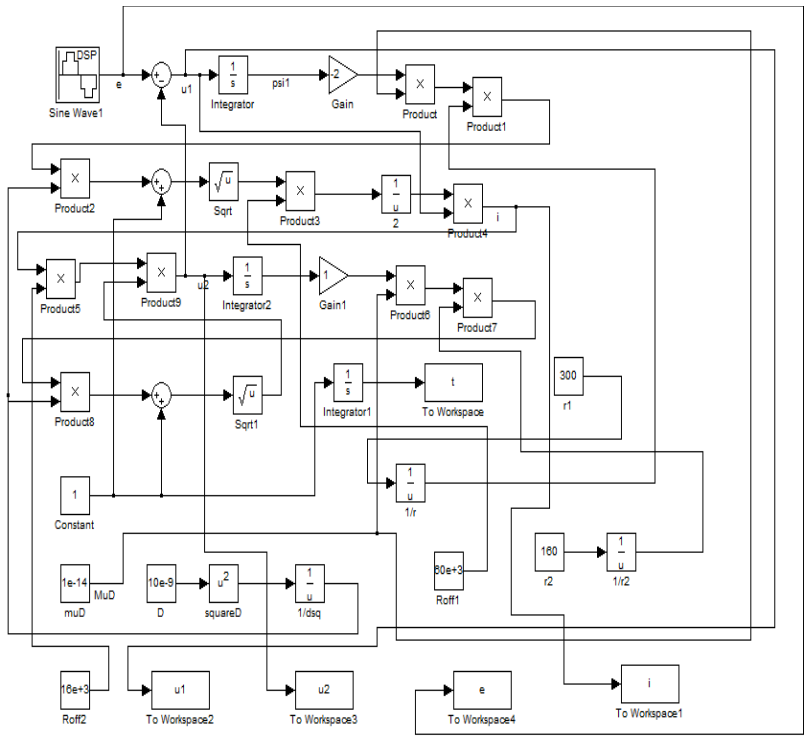

Fig. 8. Simulink model of circuit with two memristors

The relation between memristor current and voltage across $\mathrm{u}(\mathrm{t})$ is presented in [6]:

$$
i(t)=\frac{u(t)}{R_{\text {OFF }} \sqrt{\left(1-\frac{q\left(t_{0}\right)}{Q_{D}}\right)^{2}-\frac{2 \eta}{Q_{D} R_{\text {OFF }}} \int u(t) d t}}
$$

Where $\mathrm{q}\left(\mathrm{t}_{0}\right)$ is the initial charge of the memristor in the doped region and $\mathrm{Q}_{\mathrm{D}}$ is the quantity of charge in which the memristor could memorize in its whole volume [3]. Where

$$
Q_{D}=\frac{D^{2}}{\mu_{v} R_{O N}}
$$

$\mathrm{D}$ is the length of the whole memristor which is constant. The Simulink model [5] for equation (15) is shown in fig. 9.

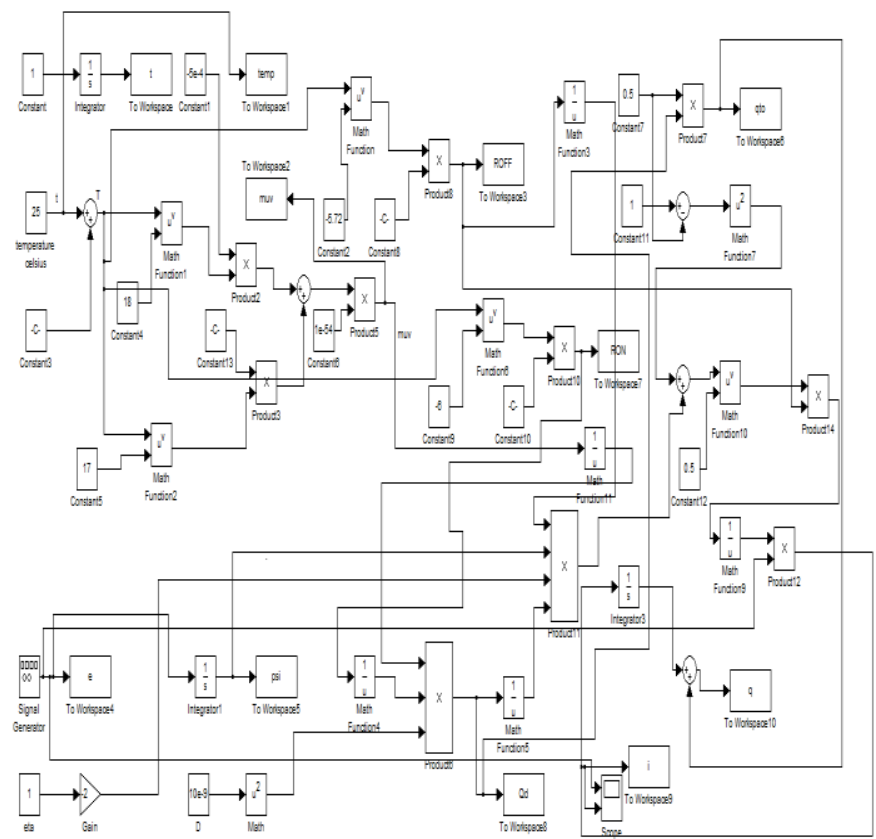

Fig. 9. Simulink model for sigle memristor with respect to temperature
Simulink model for equation (8) is shown in figure (10).

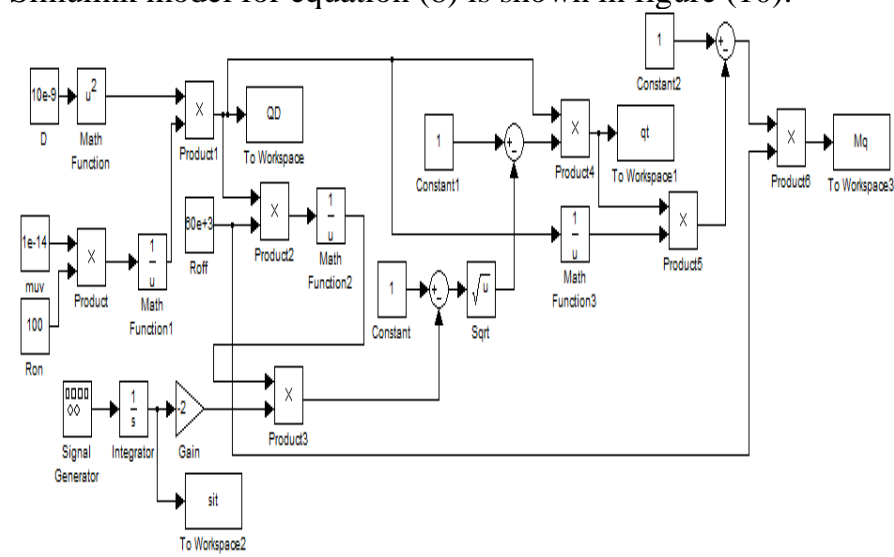

Fig 10. Simulink model for single memristor

\section{Simulation RESUlTS}

Figures 11, 12 and 13 shows that the simulation results for the single memristor as shown in fig. 4. When the sinusoidal voltage signal is applied at the input terminal with amplitude of $1 \mathrm{v}$ and frequency $1 \mathrm{~Hz}$ the EMF vs. time and current vs. time graphs are shown in figs. 11 and 12 . The volt-ampere characteristics of single memristor is given in fig. 13 .

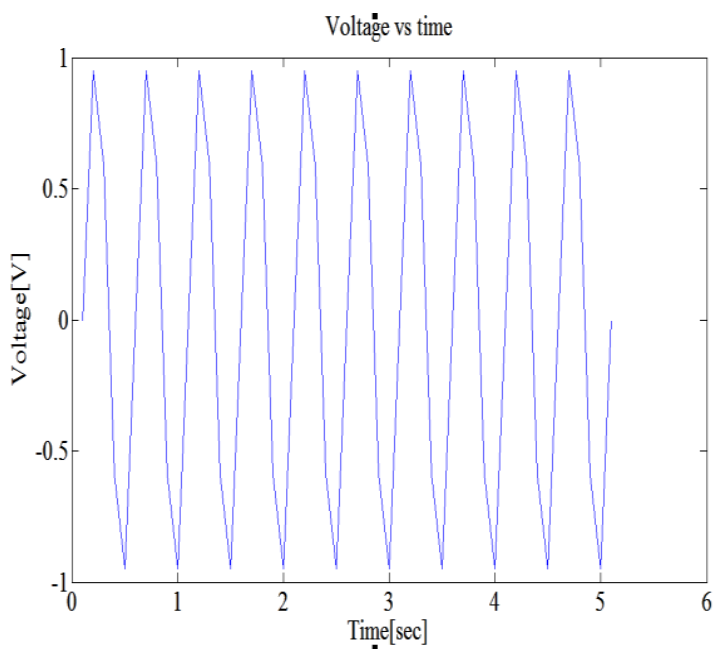

Fig. 11. Graph of voltage vs time

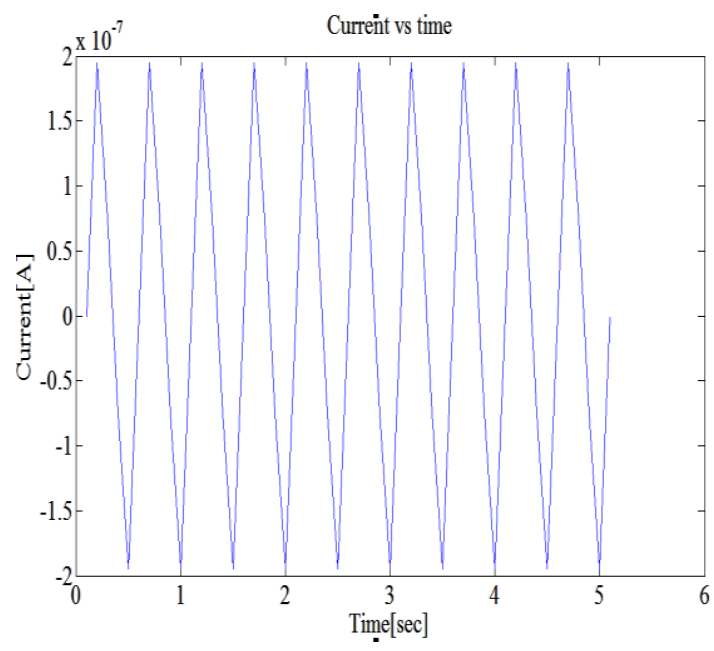

Fig. 12. Current vs time 


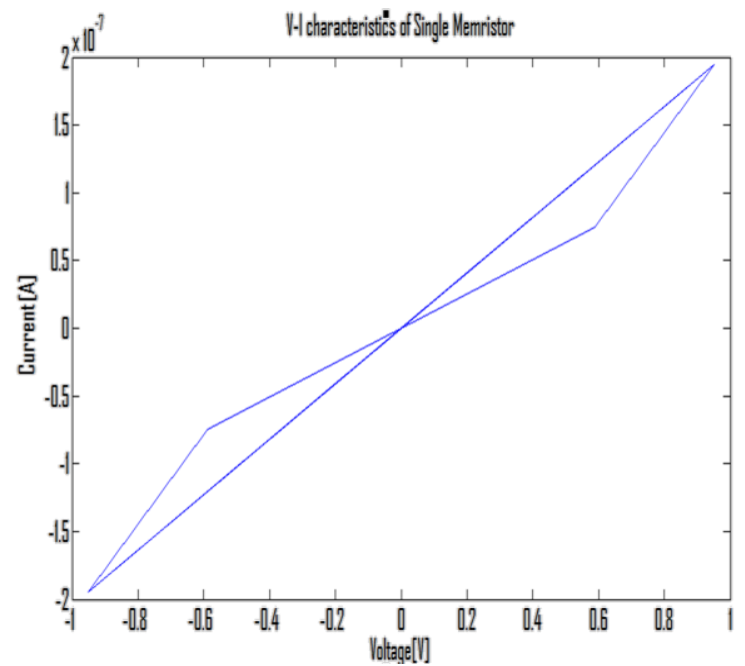

Fig. 13. V-I characteristics of single memristor

Figure 14 is the graph between on resistance and temperature in which the resistance in the closed state is decreased with increase in temperature.

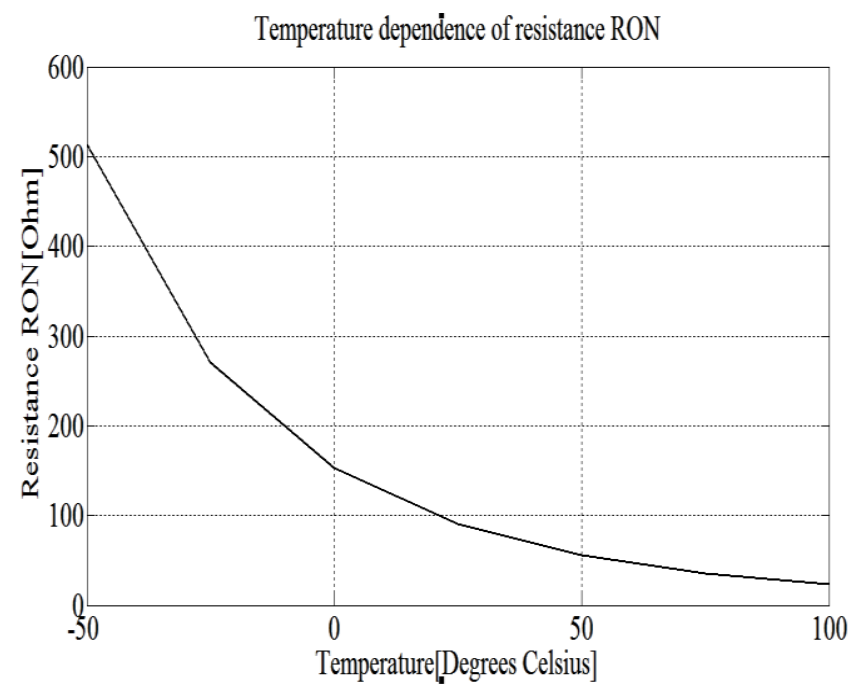

Fig. 14. Temperature vs on resistance

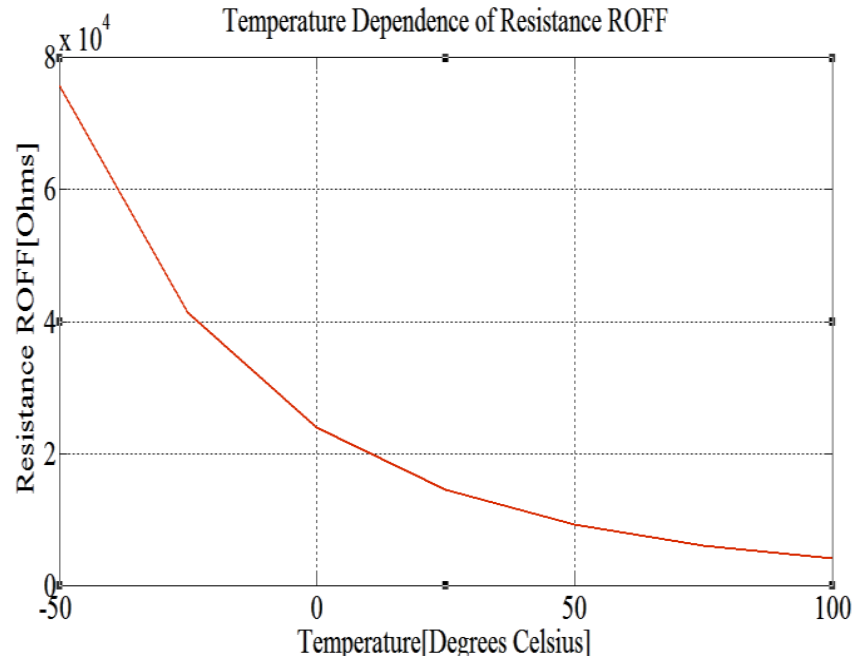

Fig. 15. Temperature vs off resistance
A graph between the reistance in open state and temperature in which the resistance is decreasing lightly with increase in temperature as shown in fig. 15.

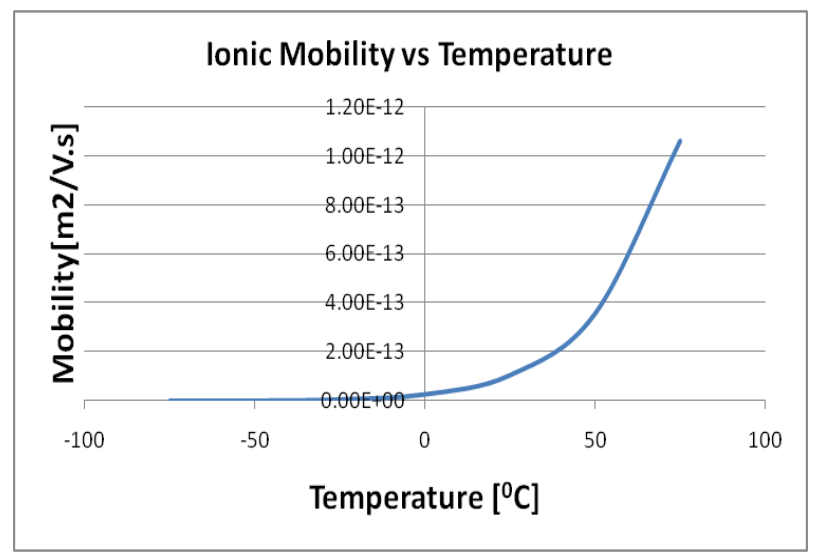

Fig. 16. Graph between charge carriers mobility and temperature

From the fig. 16 it is clear that as the memristor temperature increases rapidly, the mobility increases.

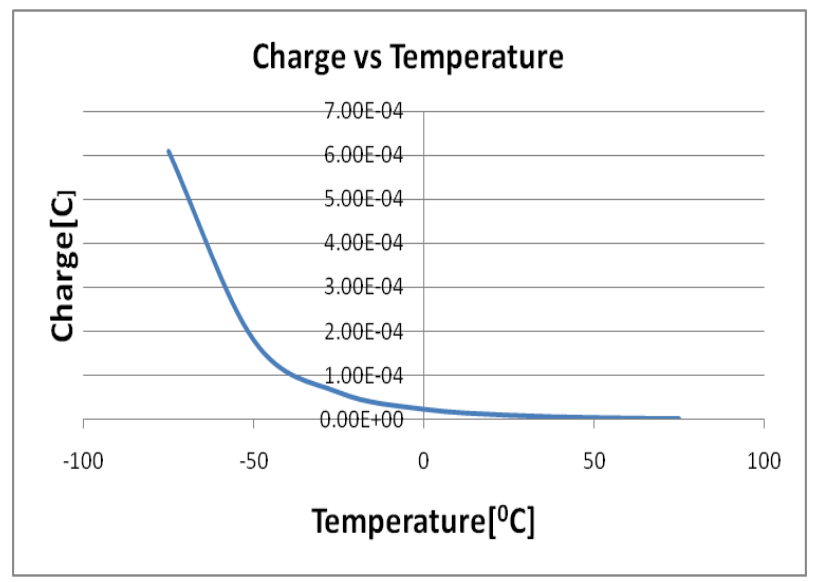

Fig. 17. Graph between charge and temperature

As the temperature increases from negative to positive, the charge decreases gradually as shown in fig. 17.

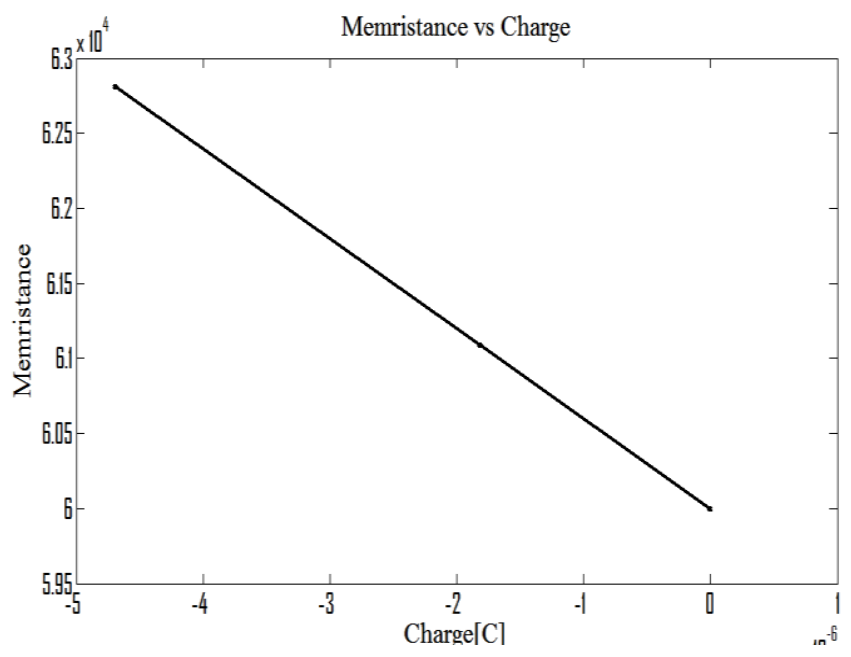

Fig. 18. Graph between charge and Memristance 
As the charge increases the memristance decreases as shown in fig. 18. A linear relationship exists between flux and memristance as given in fig. 19. In this graph as flux increases, the memristance increases linearly. The voltage vs. time graph at a magnitude of $1 \mathrm{~V}$ and frequency $1 \mathrm{~Hz}$ for the circuit 8 is shown in fig. 20. The voltage drops $\mathrm{u} 1$ and $\mathrm{u} 2$ as shown in fig. 21 and fig. 22.

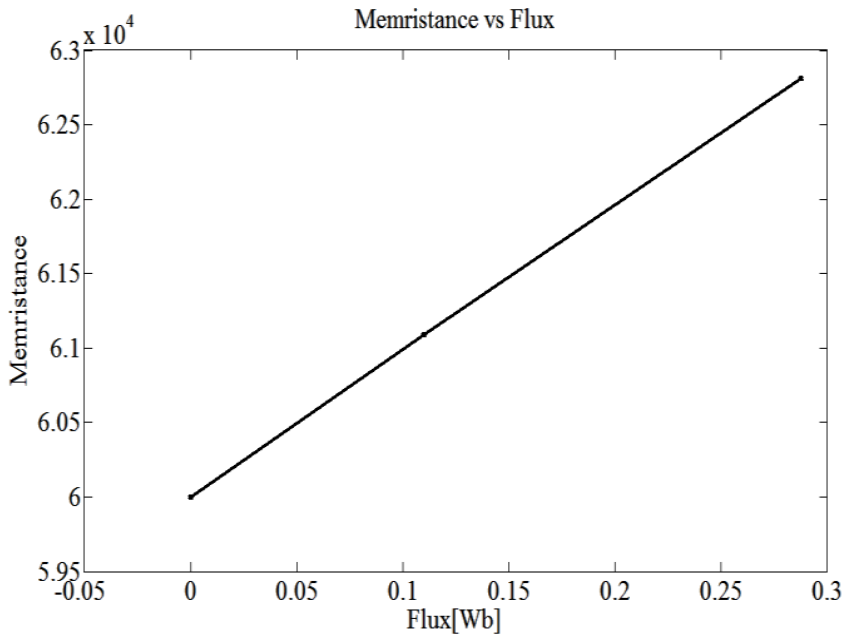

Fig. 19. Graph between Memristance and flux

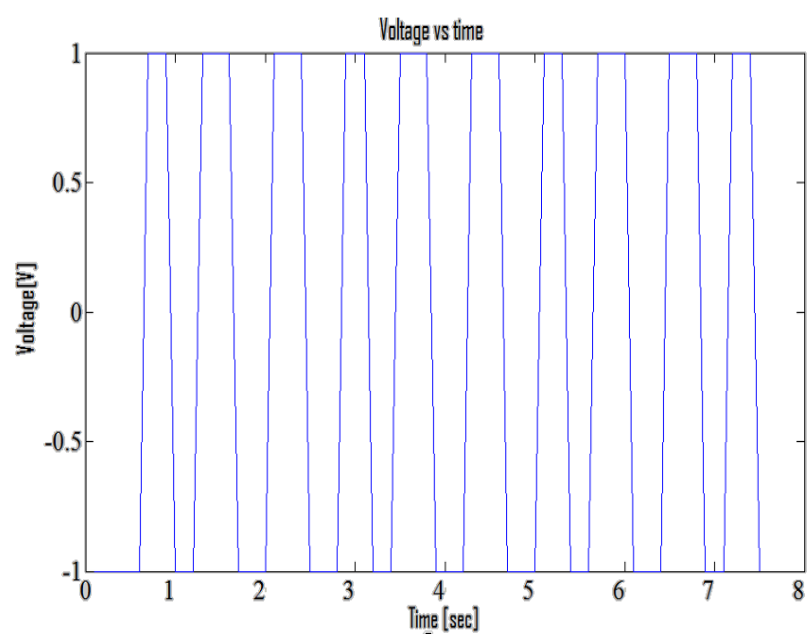

Fig. 20. Graph between EMF and time

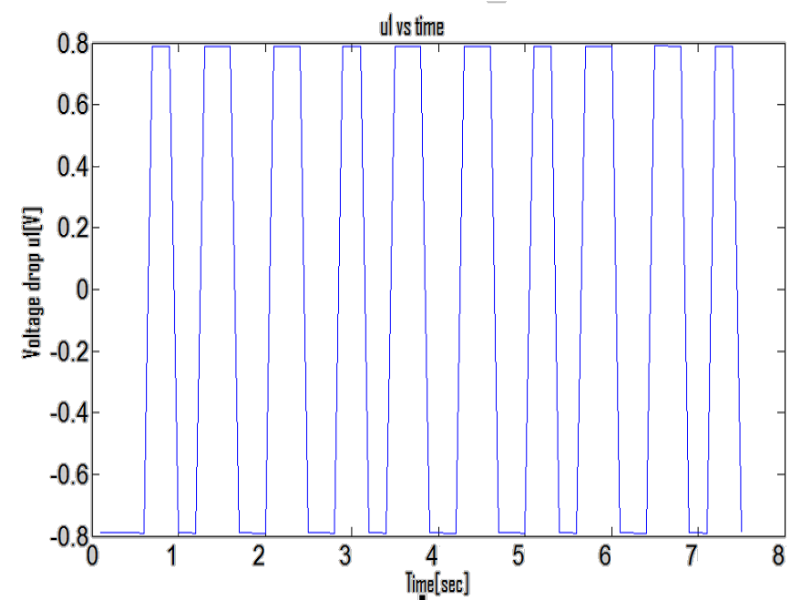

Fig. 21. Graph between voltage drop and time

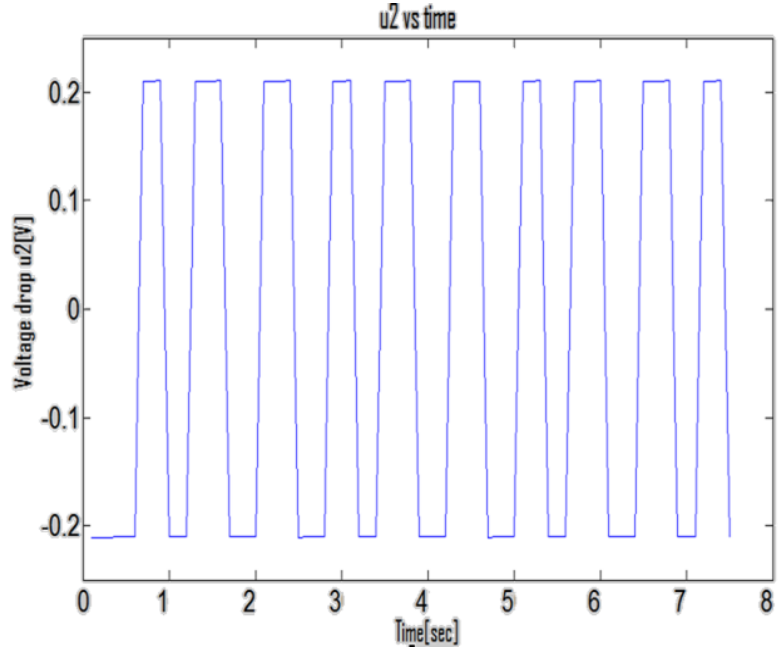

Fig.22. Graph between voltage drop and time

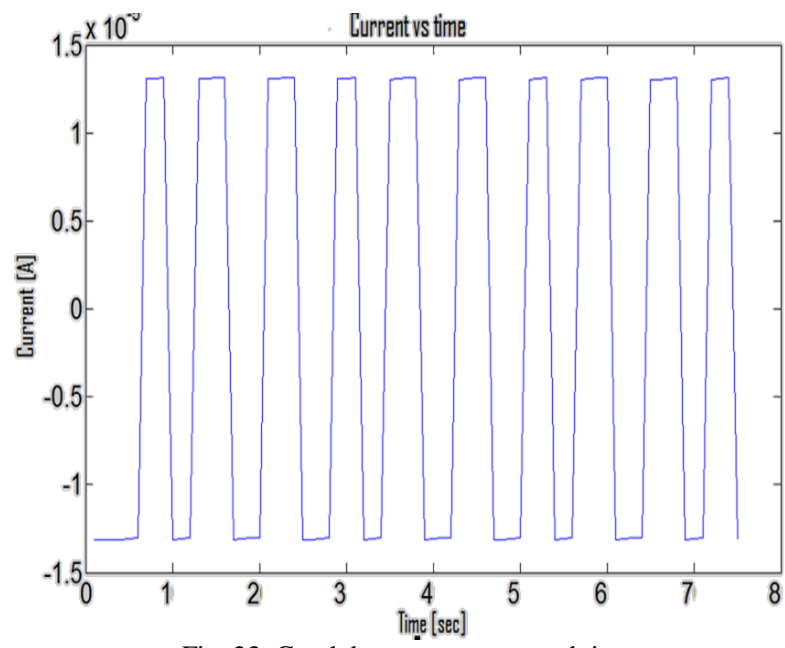

Fig. 23. Graph between current and time

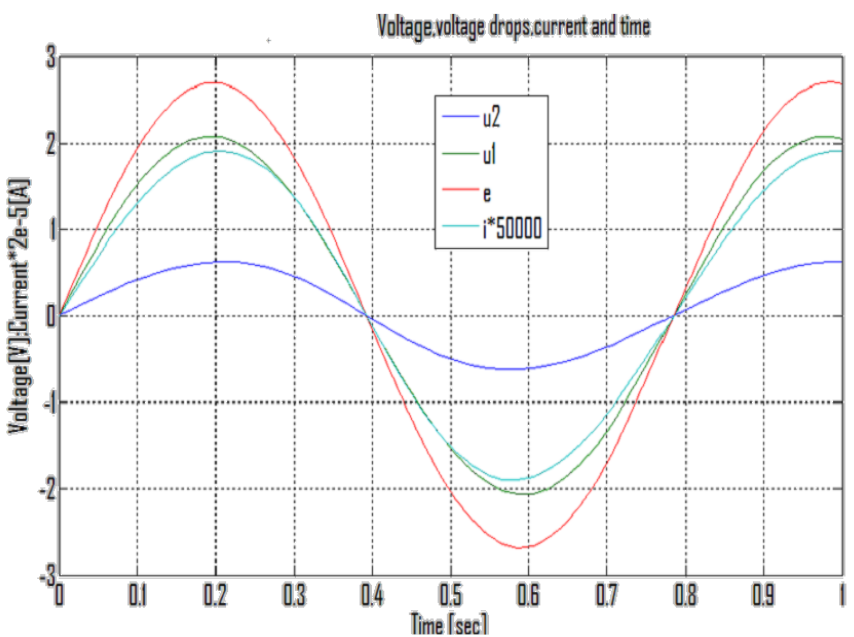

Fig. 24. Voltage, voltage drops, current vs time at $\omega=8 \mathrm{rad} / \mathrm{sec}$ and frequency $=2.7 \mathrm{v}$

the current vs. time graph at a magnitude of $1 \mathrm{~V}$ and at a a frequency of $1 \mathrm{~Hz}$ is given in fig. 23. The curve of e is ideal sinusoidal wave as shown in fig. 24. The voltage drop $u_{1}$ is higher than the voltage drop $u_{2}$. It is because of resistances of first memristor are higher than the second memristor. 


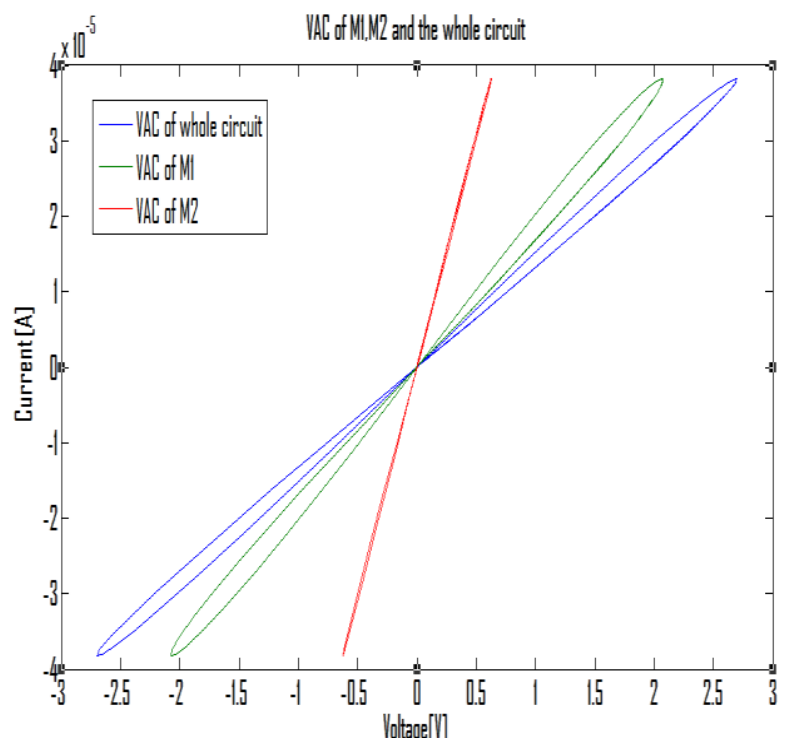

Fig. 25. Volt-ampere characteristics of memristors

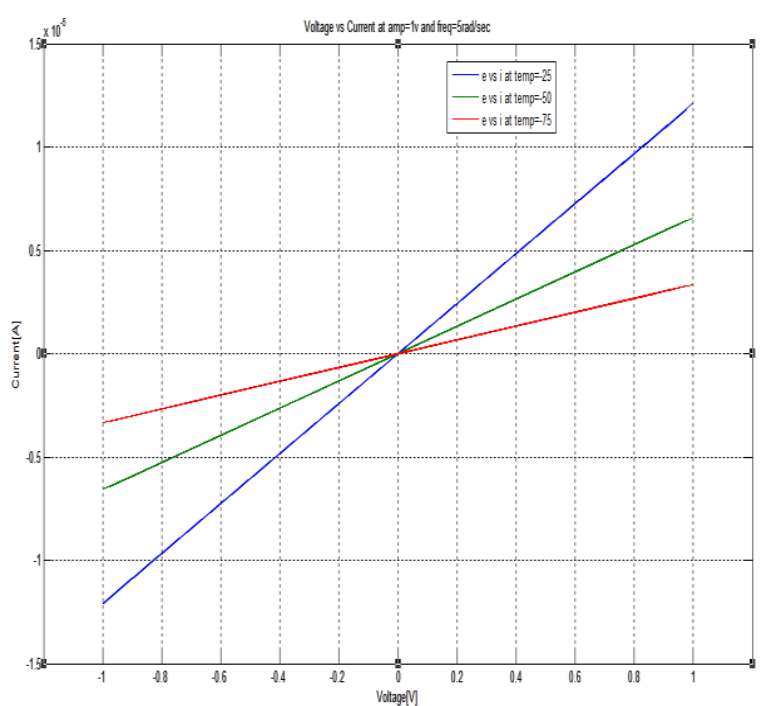

Fig. 26. Volt- ampere characteristics of memristors at different temperatures
The VI characteristics of memristor and whole circuit is given in fig. 25. The characteristics of memristors are pinched hysteresis loops. VI characteristics of $1^{\text {st }}$ memristor covers more wide area than the other memristor. The memristor M1 is more nonlinear than M2. This nonlinearity is more visible at low frequencies [2]. The VI characteristics of memristor with different temperatures is given in fig. 26.

\section{CONCLUSION}

It is clear from above results the memristor can be used as a high speed switch and it can be used in non-volatile computer memories due to its higher switching speeds. In this paper the influence of temperature on a single memristors resistance i.e., $\mathrm{R}_{\mathrm{ON}}$ and $\mathrm{R}_{\mathrm{OFF}}$ vs. temperature has been analyzed. From the results in this paper shows that as temperature increases $R_{O N}$ and ROFF decreases which will effects the switching characteristics and also its voltage-ampere characteristics. Due to the several advantages of memristors like small dimensions and high speed it can be used in neural networks, signal processing and also in combination with CMOS transistor i.e., hybrid memristor-CMOS transistor IC's. All the simulations are performed in matlab Simulink.

In this paper after complete analysis the results shows that with the increase of ambient and working temperatures of $\mathrm{TiO}_{2}$ memristor has shown a very drastic change on its properties and characteristics. For improving the memristor properties it is better to include the cooling devices.

\section{REFERENCES}

[1] L. O. Chua, "Memristor-the missing circuit element," IEEE Trans. Circuit Theory, vol. CT-18, no. 5, pp. 507-519, Sep. 1971.

[2] Williams, R. S. "How we found the missing memristor". IEEE Spectr., vol. 45, pp. $28-35,2008$.

[3] D. B. Strukov, G. S. Snider, D. R. Stewart, and R. S. Williams, "The Missing memristor found," Nature, vol. 453, no. 7191, pp. 80-83, 2008.

[4] Pazienza, G. E., J. Albo-Canals. "Teaching Memristors to EE Undergraduate Students". IEEE Circuits and Systems Magazine, pp. 36- 44, 22 November 2011.

[5] Valeri M. Mladenov, Stoyan M. Kirilov, "Analysis of a serial circuit with two Memristors and voltage source at sine and impulse regime", Technical University of Sofia, Bulgaria.

[6] Valeri M. Mladenov, Stoyan M. Kirilov, "Analysis of temperature influence on titanium dioxide memristor characteristics at pulse mode", Technical University of Sofia, Bulgaria. 